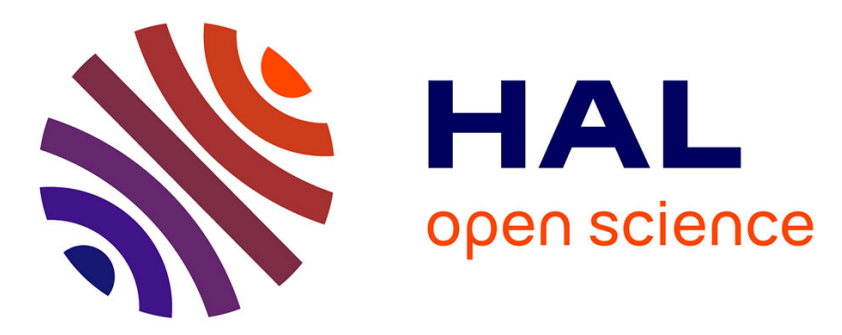

\title{
On the inverse Cauchy problem for linear ordinary differential equations
}

Maya Chartouny, Thomas Cluzeau, Alban Quadrat

\section{To cite this version:}

Maya Chartouny, Thomas Cluzeau, Alban Quadrat. On the inverse Cauchy problem for linear ordinary differential equations. GAMM 2021 - 92nd Annual Meeting of the International Association of Applied Mathematics and Mechanics, Mar 2021, Kassel, Germany. 10.1002/pamm.202100214 . hal-03530281

\section{HAL Id: hal-03530281 \\ https://hal.inria.fr/hal-03530281}

Submitted on 17 Jan 2022

HAL is a multi-disciplinary open access archive for the deposit and dissemination of scientific research documents, whether they are published or not. The documents may come from teaching and research institutions in France or abroad, or from public or private research centers.
L'archive ouverte pluridisciplinaire $\mathbf{H A L}$, est destinée au dépôt et à la diffusion de documents scientifiques de niveau recherche, publiés ou non, émanant des établissements d'enseignement et de recherche français ou étrangers, des laboratoires publics ou privés. 


\title{
On the inverse Cauchy problem for linear ordinary differential equations
}

\author{
Maya Chartouny ${ }^{1, *}$, Thomas Cluzeau ${ }^{2, * *}$, and Alban Quadrat ${ }^{1, * * *}$ \\ ${ }^{1}$ Inria Paris, Ouragan project, IMJ - PRG, Sorbonne University, France \\ ${ }^{2}$ CNRS ; XLIM UMR 7252, 87060 Limoges Cedex, France
}

\begin{abstract}
The Cauchy problem characterizes the solutions of a linear ordinary differential equation that satisfies initial conditions. In this paper, we investigate the converse problem, namely, given a function that is known to satisfy a linear ordinary differential equation of a fixed order, determine the coefficients of the ordinary differential equation and the initial conditions. The techniques used to investigate the inverse Cauchy problem come from the algebraic estimation problem introduced by Fliess and Sira-Ramírez. From the perfect observation of the solution, i.e., without external perturbation and noise corrupting it, the initial value problem can be explicitly reconstructed using only iterative indefinite integrals of the solution.
\end{abstract}

Copyright line will be provided by the publisher

\section{Introduction}

In 2003, Fliess and Sira-Ramírez proposed a new approach to parameter estimation [Fliess et al. (2003)]. This problem aims at estimating unknown constant parameters of a signal or a control system from observation which can be corrupted by perturbation and noise. Within this framework, a signal $x(\theta, t)$ is known to follow a certain linear ordinary differential equation (ODE) with unknown initial conditions. The problem aims at estimating the constant parameter system $\theta$ and the initial conditions by means of a corrupted observation $y(t)=x(\theta, t)+\gamma(t)+\varpi(t)$ of $x(\theta, t)$ and iterative indefinite integrals of $y(t)$, where $\gamma$ denotes here a structured perturbation that follows a known dynamics and $\varpi$ a noise. This approach has two main interests: the iterative integrals naturally filter the noise and non-asymptotical real-time schemes can be obtained for estimating $\theta$ [Fliess et al. (2003)]. An effective study of the algebraic estimation problem has been initiated in [Quadrat (2017)].

It is natural to ask whether or not the system parameter $\theta$ can always be estimated in the exact case, i.e., when $\gamma=0$ and $\varpi=0$. Indeed, if it is not possible, then the algebraic parameter estimation cannot be solved. Hence, in this paper, we ask whether or not the coefficients of a linear ODE with polynomial coefficients and the initial conditions of the Cauchy problem can be exactly recovered from the knowledge of a "generic solution" and of its iterative indefinite integrals. In other words, in this paper, we aim at explicitly study the inverse Cauchy problem for linear ODE with polynomial coefficients.

\section{Explicit estimation of the initial conditions}

Let us suppose that $x$ satisfies the following ODE

$$
\sum_{i=0}^{n} a_{i}(t) x^{(i)}(t)=0
$$

where the $a_{i}$ 's are polynomials in $t$ with coefficients in $\mathbb{Q}$, i.e., $a_{i}(t)=\sum_{j=0}^{d_{i}} a_{i j} t^{j}$ and $a_{i j} \in \mathbb{Q}$. Let set $m:=\max _{0 \leq i \leq n} d_{i}$.

In this section, we suppose that the coefficients $a_{i j}$ are known and we study the possibility to recover the initial conditions of the Cauchy problem for (1), i.e., the values $x^{(i)}(0)$ for $i=0, \ldots, n-1$. In Section 3, we will show how the coefficients $a_{i j}$ can be explicitly determined from $x$ and iterative indefinite integrals $\int_{0}^{t} \ldots \int_{0}^{t} x(t) d t \ldots d t$ of $x$. To do that, we use the framework of the algebraic estimation problem [Fliess et al. (2003)]. Let $\mathscr{L}$ denote the Laplace transform, namely

$$
\mathscr{L}(f)(s)=\int_{0}^{+\infty} e^{-s t} f(t) d t
$$

where $s$ is a complex number defined in a strip of $\mathbb{C}$. We recall that the Laplace transform satisfies the standard identities:

- $\mathscr{L}\left(f^{(n)}\right)(s)=s^{n} \mathscr{L}(f)(s)-\sum_{i=0}^{n-1} s^{n-i-1} f^{(i)}(0)$,

- $\mathscr{L}\left(t^{n} f\right)(s)=(-1)^{n} \partial_{s}^{n}(\mathscr{L}(f)(s))$, where $\partial_{s}^{n} g(s):=\frac{d^{n} g(s)}{d s^{n}}$ denotes the $n^{\text {th }}$ derivative of $g$ with respect to $s$.

*mayachartouny@hotmail.com

** thomas.cluzeau@unilim.fr

*** Corresponding author: alban.quadrat@inria.fr 
Hence, with the notation $\widehat{x}=\mathscr{L}(x)$, using (1), we obtain:

$$
\sum_{i=0}^{n}\left(a_{i}\left(-\partial_{s}\right) s^{i}\right) \widehat{x}(s)-\sum_{k=0}^{n-1} \sum_{i=k+1}^{n}\left(a_{i}\left(-\partial_{s}\right) s^{i-k-1}\right) x^{(k)}(0)=0
$$

For more details, see [Quadrat (2017)]. Let us note:

$$
P=\sum_{i=0}^{n} a_{i}\left(-\partial_{s}\right) s^{i}, \quad S_{k}=-\sum_{i=k+1}^{n} a_{i}\left(-\partial_{s}\right) s^{i-k-1}, \quad \vartheta_{k}=x^{(k)}(0), \quad k=0, \ldots, n-1, \quad Q=\sum_{k=0}^{n-1} S_{k} \vartheta_{k}
$$

Notice that $P$ is a OD operator in $\partial_{s}$ with polynomial coefficients in $s$, i.e., is an element of the first Weyl algebra $A_{1}(\mathbb{Q}):=$ $\mathbb{Q}[s]\left\langle\partial_{s} \mid \partial_{s} s=s \partial_{s}+1\right\rangle$ (see, e.g., [Coutinho (1995)]), which applies to the function $\widehat{x}(s)$, and in the term $S_{k}, a_{i}\left(-\partial_{s}\right) \in$ $A_{1}(\mathbb{Q})$ applies to $s^{i-k-1}$, which shows that $S_{k} \in \mathbb{Q}[s]$ and $Q \in \mathbb{Q}\left[s, \vartheta_{0}, \ldots, \vartheta_{n-1}\right]$. The above identity becomes

$$
P\left(s, \partial_{s}\right) \widehat{x}(s)+Q(s, \vartheta)=0,
$$

where we have explicitly expressed the dependence of $P$ and $Q$. Let us study when the $\vartheta_{k}$ 's can be explicitly characterized. Now, if we note $\Theta:=\left(\vartheta_{0} \ldots \vartheta_{n-1}\right)^{T}$, then (3) can be rewritten as:

$$
\left(S_{0} \ldots S_{n-1}\right) \Theta=-P \widehat{x}(s)
$$

If we denote by $v_{0}\left(a_{i}\right)$ the valuation of $a_{i} \in \mathbb{Q}[t]$ at $t=0$, i.e., the maximal power of $t$ which divises $a_{i}$, then we have:

$$
\operatorname{deg}\left(S_{k}\right)=\max _{i=k+1, \ldots, n}\left\{i-v_{0}\left(a_{i}\right)-k-1\right\}
$$

Example 2.1 If $t=0$ is a singular point for (1), i.e., $a_{n 0}=0$, then we have $a_{n}(t)=p(t) t$ for a certain polynomial $p$, and thus, $S_{n-1}=-a_{n}\left(-\partial_{s}\right) s^{0}=p\left(-\partial_{s}\right) \partial_{s} 1=0$ and $Q=\sum_{k=0}^{n-2} S_{k} \vartheta_{k}$. Hence, $\vartheta_{n-1}=x^{(n-1)}(0)$ cannot be estimated. Similarly, if $v_{0}\left(a_{n}\right) \geq 2$ and $v_{0}\left(a_{n-1}\right) \geq 1$ (e.g., $\left.t^{2} x^{(2)}(t)+t x^{(1)}(t)+x(t)=0\right)$, then $S_{n-1}=0$ and $S_{n-2}=-\left(a_{n}\left(-\partial_{s}\right) s+a_{n-1}(-\partial) 1\right)=0$, which shows that both $\vartheta_{n-1}$ and $\vartheta_{n-2}$ cannot be estimated.

In what follows, we shall suppose that $v_{0}\left(a_{n}\right)=0$, i.e., $a_{n 0} \neq 0$. Hence, $t=0$ is a regular point of (1), and thus, it makes sense to consider the Cauchy problem for (1) at $t=0$. Then, $S_{k}$ has degree $n-k-1$ in $s$ for $k=0, \ldots, n-1$.

If we differentiate $n-1$ times (4) with respect to $s$, we get $S \Theta=-\left(P \partial_{s} P \ldots \partial_{s}^{n-1} P\right)^{T} \widehat{x}(s)$, where $S$ is defined by:

$$
S=\left(\begin{array}{ccc}
S_{0} & \cdots & S_{n-1} \\
S_{0}^{\prime} & \cdots & S_{n-1}^{\prime} \\
\vdots & \vdots & \vdots \\
S_{0}^{(n-1)} & \cdots & S_{n-1}^{(n-1)}
\end{array}\right)=\left(\begin{array}{ccccc}
S_{0} & S_{1} & S_{2} & \cdots & -a_{n 0} \\
S_{0}^{\prime} & S_{1}^{\prime} & S_{2}^{\prime} & \cdots & 0 \\
\vdots & \vdots & \vdots & \cdots & \vdots \\
S_{0}^{(n-2)} & -(n-2) ! a_{n 0} & 0 & \cdots & 0 \\
-(n-1) ! a_{n 0} & 0 & 0 & \cdots & 0
\end{array}\right)
$$

The matrix $S$ is "anti-triangular" and since $a_{n 0} \neq 0$, we then get:

$$
\operatorname{det}(S)=(-1)^{\frac{n(n+1)}{2}} a_{n 0}^{n} \prod_{k=0}^{n-1} k ! \neq 0 \Longrightarrow\left(\begin{array}{c}
\vartheta_{0} \\
\vdots \\
\vartheta_{n-1}
\end{array}\right)=-S^{-1}\left(\begin{array}{c}
P \\
\partial_{s} P \\
\vdots \\
\partial_{s}^{n-1} P
\end{array}\right) \widehat{x}(s)
$$

Hence, all the $\vartheta_{i}$ 's can be explicitly expressed in terms of derivatives of $\widehat{x}(s)$ and of the $a_{i}$ 's.

Let $R$ denote the submatrix of $S$ obtained by deleting the last column and the last row of $S$. Moreover, let $\operatorname{LC}\left(p, x_{i}\right)$ denote the leading coefficient of a polynomial $p \in \mathbb{Q}\left[x_{1}, \ldots, x_{n}\right]$ with respect to the variable $x_{i}$. The inverse $\left(T_{i, j}\right)_{1 \leq i, j \leq n}$ of an upper anti-triangular matrix $S=\left(S_{i, j}\right)_{1 \leq i, j \leq n}$ can then be explicitly characterized as follows:

$$
T_{i, j}=\left\{\begin{array} { l l } 
{ T _ { i , j } = 0 , } & { i + j < n + 1 , } \\
{ \frac { N _ { i , j } } { D _ { i , j } } , } & { i + j \geq n + 1 , }
\end{array} \quad \left\{\begin{array}{rl}
D_{i, j} & =\prod_{n+1-j \leq k \leq i} S_{n+1-k, k}, \\
N_{n, n} & =(-1)^{\frac{n(n-1)}{2}} \operatorname{det}(R), \\
N_{n, j} & =-\operatorname{LC}\left(N_{n, j+1}, S_{j, n-j}\right), j=n-1, \ldots, 1, \\
N_{i, j} & =-\operatorname{LC}\left(N_{i+1, j}, S_{n-i, i}\right), i=n-1, \ldots, 1, j=n, \ldots, 1
\end{array}\right.\right.
$$


We can use the above result to compute the inverse of the matrix $S$ defined by (5), i.e., with $S_{i, j}=S_{j-1}^{(i-1)}$ where the $S_{j}$ 's are the polynomials defined by (2). Using (6), we get:

$$
\vartheta_{i}=-\sum_{j=1}^{n} T_{i, j} \partial_{s}^{j-1} P\left(s, \partial_{s}\right) \widehat{x}(s), \quad i=0, \ldots, n-1 .
$$

Note that $\operatorname{deg}_{s} \partial_{s}^{l} P=\operatorname{deg}_{s} P=n$. Moreover, using $\operatorname{deg}_{s} S_{k}=n-k-1$, we get $\operatorname{deg}_{s} S_{i, j}=n+1-i-j$ for $i+j \leq n+1$ and $S_{i, j}=0$ for $i+j>n+1$. Hence, $\operatorname{deg}_{s} S_{i, j}$ is constant on each parallel of the anti-diagonal $n+1-i-j=0$ and thus $\operatorname{deg}_{s} T_{i j}=i+j-n-1$ for $i+j \geq n+1$. Using (7), we get $\operatorname{deg}_{s} \vartheta_{i}=\max _{j=1, \ldots, n}\{i+1+j-n-1\}+n=n+i$ for $i=0, \ldots, n-1$. To get explicit expressions of the $\vartheta_{i}$ 's in terms of $x(t)$ and its iterative indefinite integrals, we rewrite the right hand side of (7), simply denoted by $r_{i}$, as the quotient of $n_{i}:=r_{i} / s^{n+i+1}$ by $d_{i}:=1 / s^{n+i+1}$, and then apply the inverse Laplace transform $\mathscr{L}^{-1}$ to get $\mathscr{L}^{-1}\left(n_{i}\right)$ and $\mathscr{L}^{-1}\left(d_{i}\right)$. Since $\vartheta_{i}$ is a constant and $\mathscr{L}^{-1}$ is a linear transformation, we obtain:

$$
\mathscr{L}^{-1}\left(\vartheta_{i} d_{i}\right)=\mathscr{L}^{-1}\left(n_{i}\right) \quad \Longrightarrow \quad \vartheta_{i}=\frac{\mathscr{L}^{-1}\left(n_{i}\right)}{\mathscr{L}^{-1}\left(d_{i}\right)}, \quad i=0, \ldots, n-1, \quad \mathscr{L}^{-1}\left(d_{i}\right)=\frac{t^{n+i}}{(n+i) !} .
$$

Finally, note that the term in the inverse Laplace transform of the right hand side of the following equation

$$
\mathscr{L}^{-1}\left(n_{i}\right)=-\sum_{j=1}^{n} \mathscr{L}^{-1}\left(\frac{T_{i, j}}{s^{n+i+1}} \partial_{s}^{j-1} P\left(s, \partial_{s}\right) \widehat{x}(s)\right), \quad i=0, \ldots, n-1,
$$

is a strictly proper rational function in $s$, namely, the degree in $s$ of its numerator is strictly less than the degree of its denominator. Hence, using the normal forms of OD operators, we have:

$$
\frac{T_{i, j}}{s^{n+i+1}} \partial_{s}^{j-1} P\left(s, \partial_{s}\right) \widehat{x}(s)=\sum_{0 \leq k \leq n+i+1,0 \leq l \leq m+j-1} \frac{c_{k l}}{s^{k}} \partial_{s}^{l} \widehat{x}(s), \quad c_{k l} \in \mathbb{Q}\left[a_{i j}\right]_{0 \leq i \leq m, 0 \leq j \leq n}
$$

Using $\mathscr{L}\left(\int_{0}^{t} y(\tau) d \tau\right)(s)=s^{-1} \widehat{y}(s)$, the inverse Laplace transform of the above right hand side shows that $\mathscr{L}^{-1}\left(n_{i}\right)$ is a finite sum of iterative indefinite integrals of terms of the form $(-t)^{l} x(t)$ - which can also be expressed as a convolution.

Example 2.2 Let $x=H_{k}$ be the $k^{\text {th }}$ Hermite polynomial satisfying $\ddot{x}(t)-2 t \dot{x}(t)+2 k x(t)=0$. Then, we have:

$$
\vartheta_{0}=x(0), \quad \vartheta_{1}=x^{\prime}(0), \quad P=2 s \partial_{s}+s^{2}+2 k+2, \quad Q=-s \vartheta_{0}-\vartheta_{1} .
$$

Hence, we get $\left(\begin{array}{lll}-s & -1\end{array}\right)\left(\begin{array}{ll}\vartheta_{0} & \vartheta_{1}\end{array}\right)^{T}=-P\left(s, \partial_{s}\right) \widehat{x}(s)$. Since $n=2$, we have to differentiate once the last equation to get:

$$
S\left(\begin{array}{l}
\vartheta_{0} \\
\vartheta_{1}
\end{array}\right)=-\left(\begin{array}{c}
P \\
\partial_{s} P
\end{array}\right) \widehat{x}(s)=-\left(\begin{array}{c}
2 s \partial_{s}+s^{2}+2 k+2 \\
\partial_{s}\left(2 s \partial_{s}+s^{2}+2 k+2\right)
\end{array}\right) \widehat{x}(s), S=\left(\begin{array}{cc}
-s & -1 \\
-1 & 0
\end{array}\right), S^{-1}=\left(\begin{array}{cc}
0 & -1 \\
-1 & s
\end{array}\right) .
$$

Hence, we have:

$$
\left(\begin{array}{l}
\vartheta_{0} \\
\vartheta_{1}
\end{array}\right)=-S^{-1}\left(\begin{array}{c}
P \\
\partial_{s} P
\end{array}\right) \widehat{x}(s)=\left(\begin{array}{c}
2 s \frac{d^{2} \widehat{x}(s)}{d s^{2}}+\left(s^{2}+2(k+2) s\right) \frac{d \widehat{x}(s)}{d s}+2 s \widehat{x}(s) \\
-2 s^{2} \frac{d^{2} \widehat{x}(s)}{d s^{2}}+\left(-s^{3}-2(k+1) s\right) \frac{d \widehat{x}(s)}{d s}+\left(-s^{2}+2(k+1)\right) \widehat{x}(s)
\end{array}\right) .
$$

Since $\operatorname{deg}_{s} \vartheta_{0}=2$, with the above notations, we have $\vartheta_{0}=n_{0} / d_{0}$, where:

$$
n_{0}=\frac{2}{s^{2}} \frac{d^{2} \widehat{x}(s)}{d s^{2}}+\left(\frac{1}{s}+2(k+2) \frac{1}{s^{2}}\right) \frac{d \widehat{x}(s)}{d s}+\frac{2}{s^{2}} \widehat{x}(s), \quad d_{0}=\frac{1}{s^{3}} .
$$

Hence, applying the inverse Laplace transform, we get $\vartheta_{0}=\mathscr{L}^{-1}\left(n_{0}\right) / \mathscr{L}^{-1}\left(d_{0}\right)$, where $\mathscr{L}^{-1}\left(d_{0}\right)=t^{2} / 2$ and:

$$
\mathscr{L}^{-1}\left(n_{0}\right)=-\int_{0}^{t} \tau x(\tau) d \tau+2 \int_{0}^{t}(t-\tau) \tau^{2} x(\tau) d \tau+2 \int_{0}^{t}(t-\tau) x(\tau) d \tau-(2+k) \int_{0}^{t}(t-\tau)^{2} \tau x(\tau) d \tau .
$$

Finally, we can do similarly for the computation of an explicit closed-form for $\vartheta_{1}$. 


\section{Explicit estimation of the coefficients of the ordinary differential equation}

In Section 2, we showed that the initial conditions $\vartheta_{i}:=x^{(i)}(0), i=0, \ldots, n-1$, of a Cauchy problem could be explicitly obtained by iterative indefinite integrals of $x(t)$. The closed-forms for the $\vartheta_{i}$ 's obtained in Section 2 depend on the constant parameters $a_{i j}$ of the ODE. We now study how the parameters $a_{i j}$ can be estimated by iterative indefinite integrals of $x(t)$.

In the frequency domain, we recall that the ODE defined by (1) is equivalently defined by (2), i.e., by (4). In Section 2 , we differentiated $(n-1)^{\text {th }}$ times (4) to get $S \Theta=-\left(P \partial_{s} P \ldots \partial_{s}^{n-1} P\right)^{T} \widehat{x}(s)$, from which we could solve for the $\vartheta_{i}$ 's. From the last row of $S$, we can easily check that the equations $\partial_{s}^{l} P\left(s, \partial_{s}\right) \widehat{x}(s)=0$ for $l \geq n$ do not depend on the $\vartheta_{i}$ 's. More precisely, we have

$$
\forall l \geq n, \quad\left(-\partial_{s}\right)^{l} P\left(s, \partial_{s}\right) \widehat{x}(s)=\left(-\partial_{s}\right)^{l} \sum_{0 \leq i \leq n, 0 \leq j \leq m} a_{i j}\left(-\partial_{s}\right)^{j} s^{i} \widehat{x}(s)=\sum_{0 \leq i \leq n, 0 \leq j \leq m}\left(-\partial_{s}\right)^{l+j} s^{i}\left(\widehat{x}(s) a_{i j}\right)=0,
$$

and $\left(-\partial_{s}\right)^{j} s^{i}=(-1)^{j} \partial_{s}^{j} s^{i}=(-1)^{j} \sum_{k=0}^{j}\left(\begin{array}{l}j \\ k\end{array}\right) \gamma_{i}(k) s^{i-k} \partial_{s}^{j-k}$, where $\gamma_{i}(k)=i ! /(i-k)$ ! for $0 \leq k \leq i$ and 0 else. Hence, if we set $a:=\left(\begin{array}{llllll}a_{00} & a_{01} & \ldots & a_{i j} & \ldots & a_{n(m-1)} \\ a_{n m}\end{array}\right)^{T}$, for $l \geq n$, we get the following system of equations:

$$
\left(\begin{array}{ccccccc}
\left(-\partial_{s}\right)^{n} & \left(-\partial_{s}\right)^{n+1} & \ldots & \left(-\partial_{s}\right)^{n+j} s^{i} & \ldots & \left(-\partial_{s}\right)^{n+m-1} s^{n} & \left(-\partial_{s}\right)^{n+m} s^{n} \\
\vdots & \vdots & \ldots & \vdots & \ldots & \vdots & \vdots \\
\left(-\partial_{s}\right)^{l} & \left(-\partial_{s}\right)^{l+1} & \ldots & \left(-\partial_{s}\right)^{l+j} s^{i} & \ldots & \left(-\partial_{s}\right)^{l+m-1} s^{n} & \left(-\partial_{s}\right)^{l+m} s^{n}
\end{array}\right) \widehat{x}(s) a=0 .
$$

Let us suppose that $a_{n m} \neq 0$, i.e., the degree of $a_{n}$ is fixed. Set $p:=n m-1$ the number of unknown $a_{i j}$ and $l:=n+p$. Let us denote by $C$ the $p \times p$ matrix defined considering all but the last column of the first matrix of (8), $c$ the last column of the first matrix of (8), and $b$ the column vector of size $p$ formed by all but the last entry of the vector $a$. Hence, (8) is equivalent to $\left(C\left(s, \partial_{s}\right) \widehat{x}(s)\right) b=-c\left(s, \partial_{s}\right) a_{n m} \widehat{x}(s)$. Hence, if $C\left(s, \partial_{s}\right) \widehat{x}(s)$ is invertible, i.e., $\operatorname{det}\left(C\left(s, \partial_{s}\right) \widehat{x}(s)\right) \neq 0$, then we get:

$$
b=-a_{n m}\left(C\left(s, \partial_{s}\right) \widehat{x}(s)\right)^{-1} c\left(s, \partial_{s}\right) \widehat{x}(s) .
$$

Thus, $a_{i j}$ can be written as a fraction $n_{i j} / d_{i j}$, where $n_{i j}$ and $d_{i j}$ are polynomials in $s$ and some derivatives of $\widehat{x}(s)$. Now, setting $q_{i j}:=\max \left\{\operatorname{deg}_{s} n_{i j}, \operatorname{deg}_{s} d_{i j}\right\}+1$ and defining $n_{i j}^{\prime}:=n_{i j} / s^{q_{i j}}$ and $d_{i j}^{\prime}:=d_{i j} / s^{q_{i j}}$, we obtain two polynomials in $s^{-1}$ and some derivatives of $\widehat{x}(s)$. Applying the inverse Laplace transform to $n_{i j}^{\prime}$ and $d_{i j}^{\prime}$, and using the fact that the inverse Laplace transform maps a product to a convolution, i.e., $\mathscr{L}^{-1}(\widehat{f}(s) \widehat{g}(s))=f \star g$, where $(f \star g)(t):=\int_{0}^{t} f(t-\tau) g(\tau) d \tau$, the $a_{i j}$ 's are then ratios of sums of iterative indefinite integrals of convolutions of terms of the form $(-t)^{\alpha} x(t)=\mathscr{L}^{-1}\left(\partial_{s}^{\alpha} \widehat{x}(s)\right)$, i.e., are ratios of two convolutions depending only on $x(t)$. The last point to investigate is when $\operatorname{det}\left(C\left(s, \partial_{s}\right) \widehat{x}(s)\right)=0$. If so, then there exists a non-zero constant vector $d:=\left(d_{00} d_{01} \ldots d_{p}\right)^{T}$ such that $C\left(s, \partial_{s}\right) \widehat{x}(s) d=0$, and thus, we get:

$$
\left(-\partial_{s}\right)^{k} \sum_{(i, j) \in \llbracket 0, \ldots, n \rrbracket \times \llbracket 0, \ldots, m \rrbracket \backslash\{(n, m)\}} d_{i j}\left(-\partial_{s}\right)^{j} s^{i} \widehat{x}(s)=0, \quad k=n, \ldots, l .
$$

Using the inverse Laplace transform, the fact that $\mathscr{L}^{-1}\left(s^{i} \widehat{x}(s)\right)=x^{(i)}(t)+\sum_{k=0}^{i-1} \delta_{0}^{(i-k-1)} x^{(k)}(0)$, where $\delta_{0}^{(e)}$ denotes the $e^{\text {th }}$ derivative of the Dirac distribution at $t=0$, and $t^{k} \delta_{0}^{(e)}=0$ for $k>e$, we obtain that $x$ then satisfies the following EDO:

$$
t^{n} \sum_{(i, j) \in \llbracket 0, \ldots, n \rrbracket \times \llbracket 0, \ldots, m \rrbracket \backslash\{(n, m)\}} d_{i j} t^{j} x^{(i)}(t)=0 .
$$

Hence, (9) holds if $x$ is a generic solution of (1), namely, a function which does not satisfy a lower order / degree EDO.

Example 3.1 If we consider $n=2$ and $m=1$, i.e., $\left(a_{21} t+a_{20}\right) x^{(2)}(t)+\left(a_{11} t+a_{10}\right) x^{(1)}(t)+\left(a_{01} t+a_{00}\right) x(t)=0$, $a_{21} \neq 0$, then all the parameters $a_{i j}$ cannot be estimated if we take $x(t)=A \sin (t)$ in (9) since it satisfies $x^{(2)}(t)+x(t)=0$, and thus, $t^{2}\left(x^{(2)}(t)+x(t)\right)=0$, which is of the form $t^{2}\left(d_{20} x^{(2)}(t)+\left(d_{11} t+d_{10}\right) x^{(1)}(t)+\left(d_{01} t+d_{00}\right) x(t)\right)=0$.

Example 3.2 If we consider again Example 2.2, using similar techniques, we can show that:

$$
k=\frac{-\int_{0}^{t} \tau^{2} x(\tau) d \tau+4 \int_{0}^{t}(t-\tau) \tau x(\tau) d \tau+2 \int_{0}^{t}(t-\tau) \tau^{3} x(\tau) d \tau-\int_{0}^{t}(t-\tau)^{2} x(\tau) d \tau-3 \int_{0}^{t}(t-\tau)^{2} \tau^{2} x(\tau) d \tau}{\int_{0}^{t}(t-\tau)^{2} \tau^{2} x(\tau) d \tau}
$$

\section{References}

[Coutinho (1995)] S. C. Coutinho, A Primer of Algebraic D-Modules, Cambridge University Press (1995).

[Fliess et al. (2003)] M. Fliess, and H. Sira-Ramírez, An algebraic framework for linear identification ESAIM Control Optim. Calc. Variat., 9, 151-168 (2003).

[Quadrat (2017)] A. Quadrat, Towards an effective study of the algebraic parameter estimation problem, Proceedings IFAC 2017 Workshop Congress, (Toulouse, 2017). NonA: A symbolic package for the algebraic parameter estimation problem, https://who.rocq.inria.fr/ Alban.Quadrat/Non-A.html. 